\title{
CAPÍTULO XIV
}

\section{FORMACIÓN POR COMPETENCIAS EN EL CONTEXTO PROVOCADO POR LA PANDEMIA DEL COVID-19}

\section{Mayte Josefina Maldonado Leal}

Licenciada. Administración. (UNERMB). Especialista en Contaduría Pública (UCLA). Magister en Recursos Humanos (UNERMB). Dra. Ciencias Gerenciales (UNEFA). Docente Universidad Nacional Experimental "Francisco Miranda" (UNEFM). Correo electrónico:mjm10402@gmail.com.

\section{Yamarú del Valle Chirinos Araque}

Doctora en Ciencias, mención Gerencia del Desarrollo Regional. Universidad Dr. Rafael Belloso Chacín, Investigadora Senior categorizada por Colciencias, docente investigadora del grupo GORAS y ECOSOL. Universidad Católica Luis Amigó Medellín, Colombia. Correo electrónico: yamaruchirinosaramigo.edu.edu.co. Registro ORCID http://orcid.org/0000-0003-0471-9859

\section{Nataliya Barbera Alvarado}

Doctora en Planificación y Gestión del Desarrollo Regional de la Universidad del Zulia. Investigador Asociado categorizado MINCIENCIAS. Docente investigador vinculado al Programa de Arquitectura, Facultad de Ciencias Humanas, Arte y Diseño, Universidad del Sinú - Elías Bechara Zainúm. Montería, Colombia. Correo electrónico: nataliaberbera@unisinu.edu.co. ORCID ID: http://orcid.org/0000-00024566-5052.

\section{Nathalie Georgina Ramírez Barbera}

Médico Cirujano, Universidad Nacional Experimental Francisco de Miranda. Especialista en Medicina Interna, Universidad Central de Venezuela. Docente vinculado al Programa de Medicina en el del Departamento de Ciencias Básicas de la Salud, Facultad de Ciencias de la Salud de la Universidad del Sinú - Elías Bechara Zainúm. Montería, Colombia. Correo electrónico: nathalieramirez@unisinu.edu.co

\section{Resumen}

El estudio centra su atención en la importancia de la formación por competencias en el contexto provocado por la pandemia COVID-19, constituyendo una alternativa que permite a las organizaciones asumir los desafíos presentes en el mundo laboral. El objetivo del artículo fue analizar la relevancia de la formación por competencias en tiempos de pandemia, a fin de reconocer las habilidades exigidas al talento humano en los actuales 
momentos. A los efectos de lograrlo, se llevó a cabo una investigación documental con un diseño documental que, buscó ampliar el conocimiento en esta materia, apoyándose en la información existente. Cabe agregar que, el interés del estudio se concentró en la interpretación y análisis de la temática, por ende, se asumió la perspectiva cualitativa. Entre sus principales reflexiones, se encuentra la necesidad de adaptarse a los nuevos requerimientos del entorno laboral. Por otro lado, la formación por sí sola no es garantía de éxito, han de existir un conjunto de medidas complementarias que, en medio de la incertidumbre apoyen a las empresas y sus trabajadores.

Palabras clave: COVID-19, formación por competencias, organizaciones.

\begin{abstract}
The study focuses on the importance of skills training in the context caused by the COVID-19 pandemic, constituting an alternative that allows organizations to take on the challenges present in the world of work. The aim of the article was to analyze the relevance of skills training in times of pandemic, in order to recognize the skills required of human talent at the present time. In order to achieve this, a documentary investigation was carried out with a documentary design that sought to expand knowledge in this matter, relying on existing information. It should be added that the interest of the study was concentrated in the interpretation and analysis of the subject, therefore, the qualitative perspective was assumed. Among his main reflections, is the need to adapt to the new requirements of the work environment. On the other hand, training alone is not a guarantee of success, there must be a set of complementary measures that, amid the uncertainty, support companies and their workers
\end{abstract}

Keywords: COVID-19, organizations, training by competition.

\title{
Introducción
}

Ante la propagación del COVID-19 en el mundo, los países han adoptado medidas de emergencias, con el propósito de evitar el contagio, restringiendo la movilidad, exigiendo el confinamiento domiciliario, entre otras, lo cual ha repercutido en el contexto laboral. En tales escenarios, surgen nuevos requerimientos que han llevado a las organizaciones a preparar su talento humano, de tal modo que puedan enfrentar los desafíos y retos actuales. Por tal razón, la atención se centra en la formación por competencias, la cual debe estar acorde a los nuevos tiempos.

En este sentido, se exige a la gerencia una nueva manera de abordar las realidades, pues, el entorno ha cambiado drásticamente, sacudiendo las estructuras tradicionales a todo nivel. Entonces, surgen interrogantes ¿Qué papel asume el talento humano en las empresas? ¿Cómo prepararlo ante estos escenarios? ¿Cómo la gerencia elevaría el potencial del talento humano? Ante la incertidumbre, no existe una receta o una fórmula que nos dé la solución; pero la creatividad y preparación suelen ser útiles en momentos de crisis. 
En tales escenarios, la formación por competencias puede ser una opción, por ende, las empresas han de invertir en esta materia, debido a que proporcionaría un rendimiento laboral. Al respecto, Cejas et al. (2019) manifiesta los beneficios derivados de la adquisición y desarrollo de las competencias del talento humano, por cuanto mejoran el desempeño de los trabajadores y contribuyen con las metas de la organización y/o institución.

Bajo esta óptica, se expone el presente artículo, cuyo propósito es analizar la relevancia de la formación por competencias en tiempos del COVID-19, a fin de reconocer las habilidades exigidas al talento humano en el ámbito laboral. Por otra parte, el estudio genera un aporte científico; ya que se obtiene un nuevo conocimiento del proceso formativo que, permite a las organizaciones aminorar los impactos del COVID-19 en el mundo del trabajo, mediante un talento humano idóneo. De este modo, la gerencia está llamada a repensar sus estrategias y reajustar sus esquemas ante las transformaciones en el escenario socio laboral.

\section{Fundamentación teórica}

\section{Formación por competencias y su concepción}

En el campo organizacional, la formación por competencia es de gran relevancia, ya que ésta contribuye al desarrollo del talento humano, mediante la adquisición de habilidades, necesarias para un mejor rendimiento en el puesto de trabajo. Es así como Zapata (2015), manifiesta que este tipo de formación proporciona flexibilidad, multidisciplinariedad, integralidad, el encuentro entre el sector educativo y el productivo, la comunicación entre empleador y empleado, una preparación ética, entre otros. Sin embargo, el sistema educativo se encontraría al servicio del modelo económico, por lo que la educación se instrumentaliza y se vuelve utilitaria.

Por su parte, Cejas (2008) señala que este enfoque constituye un elemento clave del éxito competitivo que permite el mejoramiento del desempeño de las personas a través del desarrollo de las competencias. En este debate, conviene resaltar las acepciones del término, partiendo de las perspectivas de investigadores, a fin de apreciar las contribuciones en la materia. En tal sentido, se presenta el siguiente cuadro: 


\section{Cuadro 1}

Concepciones de la Formación por Competencias.

\begin{tabular}{cl}
\hline Autor & \multicolumn{1}{c}{ Descripción } \\
\hline $\begin{array}{c}\text { Cejas et al. } \\
(2019)\end{array}$ & $\begin{array}{l}\text { Es un proceso de enseñanza y aprendizaje que está orientado a que las personas } \\
\text { adquieran habilidades, conocimientos y destrezas empleando procedimientos o } \\
\text { actitudes necesarias para mejorar su desempeño y alcanzar los fines de la organización. }\end{array}$ \\
\hline $\begin{array}{c}\text { Alles } \\
(2017)\end{array}$ & $\begin{array}{l}\text { La formación por competencias puede darse en las etapas iníciales, no se destina a } \\
\text { personas que están ingresando a la empresa sino a aquellas que ya ocupan un puesto } \\
\text { en ellas, con el objetivo de hacerlas eficaces. La formación por competencias es la que } \\
\text { acompaña a una persona en su crecimiento. }\end{array}$ \\
\hline Tobon & $\begin{array}{l}\text { Aquella acción de suministrar a una persona o grupo, información y entrenamiento } \\
\text { preciso para conocer-aprender a realizar y desempeñar una determinada actividad laboral. }\end{array}$ \\
\hline Martínez y y desarrollar un proceso de enseñanza aprendizaje asentado en el aprender a aprender \\
Echeverría \\
$\begin{array}{l}\text { (2009) } \\
\text { yorientado a la movilización de los cuatro saberes fundamentales en situaciones reales, } \\
\text { por lo cual es difícil incrementarlos sin una estrecha relación con el entorno donde las } \\
\text { personas se desenvuelven habitualmente. }\end{array}$ \\
\hline $\begin{array}{c}\text { Márquez } \\
\text { y Díaz } \\
\text { (2005) }\end{array}$ & $\begin{array}{l}\text { Es aquella que está orientada principalmente a la producción y al desarrollo de } \\
\text { perfil de competencias para luego establecer las competencias que posee la persona } \\
\text { en un cargo, las cuales son comparadas con las competencias reales, este resultado } \\
\text { determinará la dirección a seguir en el proceso de formación. }\end{array}$ \\
\hline Valverde \\
(2001)
\end{tabular} \begin{tabular}{l}
$\begin{array}{l}\text { Permite que haya una relación directa entre las competencias requeridas y los contenidos } \\
\text { de los programas de formación. De esta manera, quienes ejecuten la formación tendrán } \\
\text { un referente para adecuar sus programas y quienes demanden sus servicios tendrán la } \\
\text { seguridad que se adaptan a las necesidades. }\end{array}$ \\
\hline
\end{tabular}

Fuente: Elaboración propia (2020).

Las visiones de estos autores vienen a enriquecer el campo de la gerencia del talento humano, puesto que proporcionan las luces que guiarán su actuación, en aras de obtener un mejor desempeño. De este modo, se tienen unos elementos que integran el proceso de formación basado en competencias, entre ellos se encuentran la identificación de la competencia que se requiere en el entorno laboral, la cual se plasmará en el perfil por competencias y en el programa o plan de formación. De igual manera, han de considerarse los métodos y procedimientos que contribuirán a mejorar el desempeño, sin perder de vista los saberes, es decir, saber ser, saber hacer y saber estar. Entonces, el desarrollo de la competencia contemplará las habilidades, el conocimiento y la acción que es movilizada por el compromiso y los valores.

Cabe destacar que, las competencias deben ir acompañadas del sentido de compromiso y la acción, tal como lo plantea Alles (2017), si un profesional tiene el sentido del compromiso y actúa; pero no dispone de las capacidades, evidentemente no alcanzará buenos resultados. Por el contrario, si posee las capacidades y actúa, sin comprometerse con el proyecto, puede lograr lo fijado; sin embargo la falta de motivación le impedirán innovar. Es importante aclarar que, cuando el talento humano posee las competencias y compromisos; pero actúa cuando ya ha pasado el momento, tampoco logrará los objetivos. Lo anterior, se ilustra en la figura 1. 


\section{Figura 1}

Gestión del Talento Humano.

$$
\text { Talento Individual }=\text { capacidades }+ \text { compromiso }+ \text { acción }
$$

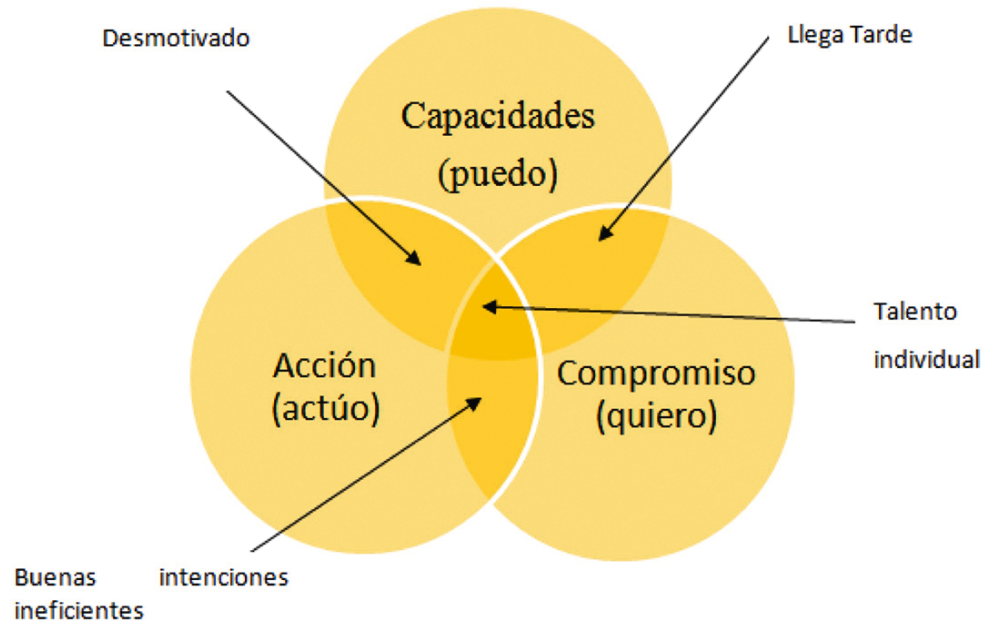

Fuente: Alles (2017).

Tal como se observa en la figura 1, el desarrollo del talento humano conjuga tres factores a saber: capacidades, compromiso y acción que, contribuyen con el adecuado desempeño y el alcance de los resultados establecidos. Sin embargo, la formación sobre la base de la competencia es compleja, por cuanto requiere la mezcla de estos atributos que van más allá de la ejecución satisfactoria del trabajo; sino que implica mirar al ser humano en toda su dimensión, tanto en sus conocimientos intelectuales, destrezas, inteligencia emocional, valores, ética, sentimientos, entre otros.

\section{Perfil por competencias y sus aportes al proceso formativo}

La formación por competencias y el perfil se encuentran estrechamente relacionados, ya que caminan de la mano para obtener el talento humano idóneo en las organizaciones. Al respecto, Márquez y Díaz (2005) consideran que la formación va dirigido principalmente a:

...la producción y al desarrollo de competencias de personas, cuyo proceso se inicia básicamente con la construcción del perfil de competencias para luego establecer las competencias que posee la persona en un cargo, las cuales son comparadas con las competencias reales, este resultado determinará la dirección a seguir en el proceso de formación. (p.97) 
De acuerdo a esta perspectiva, el proceso formativo tiene como punto de partida el reconocimiento del desarrollo de la competencia, soportado en el diseño del perfil, el cual proporcionará los lineamientos que guiarán la formación. Por tanto, aporta información que facilita la elaboración del plan o programa, donde se plasmarán las acciones y estrategias que conducirán al logro de la competencia deseada en el contexto laboral. En tal sentido, Vela (2004) señala que el perfil contribuye en cada momento con la obtención de las competencias requeridas por las organizaciones, por cuanto permite a las empresas adaptarse a las necesidades. Ahora bien, la formación se apoya en un proceso que consta de unas fases, las cuales se ilustran en la figura 2.

\section{Figura 2}

Formación por Competencias.

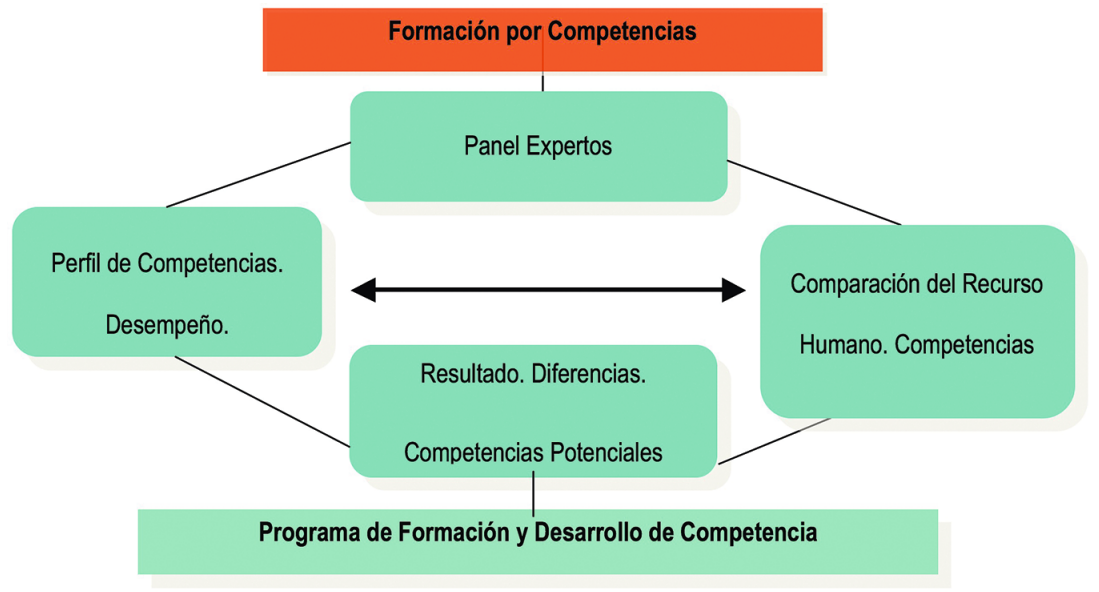

Fuente: elaboración propia (2020).

En la figura anterior, se observa que el proceso inicia con la investigación sobre la organización y los cargos, cuya responsabilidad recaerá en un grupo de expertos, quienes tendrán en cuenta la misión, visión, valores de la organización, entre otros, con base a estos datos se procederán a determinar las competencias para ocupar un cargo específico. Al respecto, se efectuarán entrevistas para conocer las habilidades existentes, centrándose en las establecidas en el perfil de la empresa, posteriormente, se realizará una comparación, con el propósito de encontrar las brechas entre la competencia solicitada y la realidad encontrada en el campo laboral, constituyendo el fundamento que permite la construcción del plan o programa de formación, el cual tiene como fin el aprovechamiento de las potencialidades del talento humano y adecuación a los cargos.

Resulta propicio mencionar a Alles (2007), quien indica que el diseño del perfil por competencias se apoya en la descripción del puesto, proporcionando información valiosa 
sobre las tareas, habilidades, conocimientos, entre otros. Además, desagrega la competencia en elementos más detallados, es decir, en diferentes niveles o grados, denominados A, B, C y $\mathrm{D}$, representando el valor $\mathrm{A}$ al nivel superior, $\mathrm{B}$ y $\mathrm{C}$ a los intermedios y $\mathrm{D}$ como el mínimo de la competencia. Los aspectos mencionados son llenados en un formulario, donde cada empresa los elabora en función de sus necesidades, naturaleza del trabajo, misión, visión, valores, entre otros. De este modo, el perfil del puesto por competencias se adapta a la organización, constituyendo de cierta forma un sello distintivo. Agrega la autora que, al definir el perfil se tomará en cuenta:

- Datos sobre la educación y experiencia laboral.

- Establecer la competencia y la relación dentro de la organización, así como también se debe reconocer la estructura jerárquica, revisar el organigrama, observar las conexiones del cargo, desempeño, entre otros.

- La remuneración asignada a un puesto de trabajo es un dato relevante.

- Esbozar el plan de carrera del candidato a seleccionar, el cual se recomienda dejar para el final del proceso.

En atención a lo expresado anteriormente, se tiene que al diseñar el perfil, la gerencia contemplará los datos de la organización y del puesto de trabajo, extrayendo cuidadosamente la información, con el fin de construirlos en forma eficaz y eficiente. Es importante mencionar que cada cargo, puede contener diferentes características y competencias, dependiendo de la naturaleza del trabajo a realizar. Sobre estas consideraciones, Maldonado (2012) esquematiza en la figura 3, los pasos empleados para elaborar el perfil por competencias, el cual aplicó en el departamento de presupuesto de la Universidad Nacional Experimental "Francisco de Miranda" (UNEFM), ubicada en el Estado Falcón en Venezuela.

\section{Figura 3}

Diseño del Perfil por Competencias.

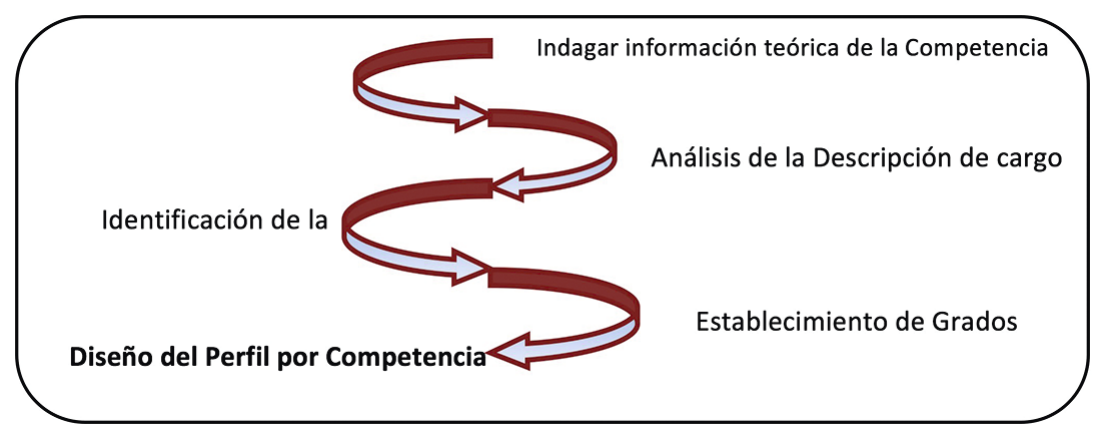

Fuente: Maldonado (2012). 
En la figura que antecede, se visualiza que el diseño del perfil parte fundamentalmente de las investigaciones sobre las teorías existentes, luego se consideran las descripciones de los cargos que revelan los contenidos del puesto de trabajo. De igual manera, se procede a identificar las competencias, es decir, las habilidades y conocimientos requeridos en el cargo, teniendo presente el establecimiento de los grados, siendo A el de mayor nivel y D el mínimo, según la teoría de Alles. Conviene mencionar que la identificación de la competencia es clave en la construcción del perfil, por ende, debemos tener claridad en la tipología de las competencias (ver cuadro 2), el entorno interno y externo de la empresa, el cargo, entre otros.

\section{Cuadro 2}

\section{Clasificación de las Competencias}

\begin{tabular}{cl}
\hline Tipos & \multicolumn{1}{c}{ Detalle } \\
\hline \multirow{3}{*}{ Cardinales } & $\begin{array}{l}\text { Aquellas que una empresa desea que todo su personal las posea y desarrolle. Se } \\
\text { esfuerzan en desarrollar estas capacidades, contribuyen con el logro de sus metas } \\
\text { y objetivos }\end{array}$ \\
\hline \multirow{3}{*}{ Específicas } & $\begin{array}{l}\text { Presentan directa relación con un puesto o familia de puestos. Tienen un rango de } \\
\text { grados, representado en cuatro niveles, en los que la letra A corresponde al mayor } \\
\text { contenido y la letra D las de menor contenido. }\end{array}$ \\
$\begin{array}{l}\text { Ejecutivas. Están relacionadas con la alta gerencia, ocupando cargos estratégicos. } \\
\text { - Niveles intermedios. Se asocian a los niveles medios en la estructura de la } \\
\text { organización, y son aquellas personas que poseen conocimientos y experiencia } \\
\text { laboral sin ocupar posiciones supervisoras. }\end{array}$ \\
$\begin{array}{l}\text { - Niveles Iniciales. Son trabajadores sin experiencia laboral, que se están iniciando } \\
\text { en el trabajo. }\end{array}$ \\
\hline Conocimientos \\
$\begin{array}{l}\text { Dependen principalmente del conocimiento, siendo útiles para efectuar un análisis, } \\
\text { solución de problemas, dichas competencias se requieren en toda la organización. }\end{array}$ \\
\hline
\end{tabular}

Fuente: adaptado de Alles (2017).

La tipología de las competencias tendrá en consideración la construcción del perfil, puesto que reflejará el conjunto de habilidades demandadas en el contexto laboral. En el caso de las cardinales, se exigirá que éstas sean comunes en el ambiente de trabajo. Mientras que, las específicas estarán en función del cargo que ocupe en la empresa, es decir, se encuentran determinadas por su nivel de jerarquía. Y las competencias de conocimientos, tal como su nombre lo indica se centran en el conocimiento que da valor a la organización. Para una mayor comprensión, en el cuadro 3, se presenta uno de los perfiles por competencias elaborados para el departamento de presupuesto de la UNEFM. 


\section{Cuadro 3}

Perfil por competencias del Departamento de Presupuesto UNEFM

\begin{tabular}{ccc}
\hline \multicolumn{3}{c}{ Perfil de Competencia } \\
\hline Cargo: Analista Central de Presupuesto. Adscrito a: Departamento Presupuesto \\
\hline A continuación se indican los grados requeridos (A, B, C, D) para las competencias \\
mencionadas
\end{tabular}

Experiencia: 6 años de experiencia de carácter operativo, supervisorio y estratégico en formulación, análisis y planificación presupuestaria.

Fuente: Maldonado (2012)

\section{Formación por competencias: una alternativa para las empresas en el contexto del} COVID-19.

El mundo se encuentra envuelto en ambientes turbulentos e inciertos, producto de los efectos del COVID-19, impactando dramáticamente la salud, el ámbito social con nuevas formas de relacionarnos, en el contexto económico con empresas cerradas, economías paralizadas, desempleo, entre otros. Según la Organización Internacional del Trabajo (OIT) (2020a) la pandemia tendrá amplia repercusión en el mercado laboral, estimando un aumento del desempleo a nivel mundial que oscila entre 5,3 millones (caso más favorable) y 24,7 millones de personas (caso más desfavorable), con respecto a un valor de referencia de 188 millones de desempleados en el año 2019.

Por su parte, el Consejo Mundial para el Comercio y Turismo (como se citó en la OIT 2020a) prevé una disminución de desplazamientos internacionales de hasta el $25 \%$ en 2020 , lo que podría poner en riesgo millones de puestos de trabajo. Esta reducción de la actividad económica y restricciones en materia de circulación afectará al sector transporte, servicios, turismo, entre 
otros. En tal sentido, la Comisión Económica para América Latina y el Caribe (CEPAL) (2020) manifiesta la incidencia en la productividad de los sectores económicos, fijando tres grupos con pesos muy diferentes en el producto interno bruto (PIB); entre ellos destacan:

i) Los menos afectados: agricultura, ganadería, caza, silvicultura y pesca.

ii) Los medianamente afectados: explotación de minas y canteras, industrias manufactureras, suministro de electricidad, gas y agua, construcción, intermediación financiera, inmobiliarias, servicios empresariales y de alquiler, administración pública, servicios sociales y personales.

iii) Los más afectados: comercio al por mayor y al por menor, reparación de bienes, hoteles y restaurantes, transporte, almacenamiento y comunicaciones, servicios en general. (p.12)

Significa que, la acción empresarial a nivel mundial se encuentra afectada, teniendo repercusiones en la fuerza laboral. Esta situación sin precedentes ha conducido a las organizaciones a un desequilibrio, planteándose la imperiosa necesidad de adaptarse a los cambios. Ante los nuevos escenarios, surgen inquietudes ¿Cuáles son las nuevas formas de abordar el ámbito laboral? ¿Qué competencias demandan las organizaciones de su talento humano?, ¿Cuáles serían las potencialidades del talento humano que se desarrollarían en un proceso de formación por competencias? La formación basada en competencias juega un papel determinante para las empresas, ya que se convierte en una alternativa viable que les proporcionaría el talento humano eficiente y efectivo, disminuyendo el riesgo de ser rebasados por el estado de emergencia en materia de salud, ocasionado por la pandemia. Por ende, la formación constituye una opción estratégica que facilitaría a las organizaciones su permanencia y supervivencia en los mercados, mediante la potenciación del capital humano.

En este marco de análisis, las competencias demandadas están centradas en la flexibilidad, capacidad para adaptación, responsabilidad, manejo de las tecnologías de información y comunicación (TIC), habilidades en la planificación y organización, liderazgo, entre otros. Para la empresa Deloitte (2020), se requiere tomar decisiones sobre los trabajos a distancias, aplicar estrategias virtuales, determinar la cantidad de espacio requerido por empleado en las instalaciones, entre otros. En tal sentido, las organizaciones han de formar al personal en competencias, atendiendo a las necesidades actuales, con el fin de salir airosos en medio de la incertidumbre. A fin de lograr una mayor comprensión, se presenta el cuadro 4, donde se sintetizan las visiones de investigadores sobre el tópico.

En el cuadro que antecede, los autores Aguerrevere et al. (2020) plantean que ante las nuevas formas de trabajo originadas por el COVID-19, se deben desarrollar cuatro habilidades, las primeras comprenden las destrezas digitales, empleadas en la ejecución de las tareas en ambientes virtuales, las segundas son cognitivas, asociadas al pensamiento crítico, manejo de conflictos.

Las terceras son destrezas de función ejecutiva, tales como la concentración, organización, reflexión, entre otros y finalmente, se encuentran las destrezas emocionales, relacionadas con cualidades personales, actitudes, creencias y comportamiento del individuo, por ejemplo autoestima, perseverancia, creatividad, resiliencia, manejo de emociones, entre otros. Cabe agregar que, el desarrollo de estas habilidades conduciría a la adaptación del personal a las actuales circunstancias. 


\section{Cuadro 4}

Competencias en el Contexto de la COVID-19.

\begin{tabular}{|c|c|c|}
\hline Autor & $\begin{array}{c}\text { Competencias } \\
\text { Requeridas }\end{array}$ & Descripción \\
\hline \multirow{4}{*}{$\begin{array}{l}\text { Aguerrevere, } \\
\text { et al. }(2020)\end{array}$} & Digitales & Usan la tecnología en la ejecución de las tareas. \\
\hline & Cognitivas & $\begin{array}{l}\text { Son generadoras de conocimiento. Emplean el pensamiento } \\
\text { crítico o resolución de problemas, manejo de conflictos. }\end{array}$ \\
\hline & Función Ejecutiva & $\begin{array}{l}\text { Habilidades mentales que incluyen la memoria funcional, el } \\
\text { pensamiento flexible y el autocontrol. Utilizadas para aprender } \\
\text { y trabajar. }\end{array}$ \\
\hline & Socioemocionales & $\begin{array}{l}\text { Asociadas a cualidades personales, actitudes, creencias, rasgos } \\
\text { de la personalidad y comportamiento de un individuo. }\end{array}$ \\
\hline \multirow{6}{*}{$\begin{array}{l}\text { Peiró y Soler } \\
\quad(2020)\end{array}$} & $\begin{array}{c}\text { Campo } \\
\text { Profesional } \\
\end{array}$ & $\begin{array}{l}\text { Desarrollo de destrezas propias de su perfil profesional } \\
\text { (administradores, contadores, ingenieros, entre otros). }\end{array}$ \\
\hline & \multirow{5}{*}{ Digitales } & $\begin{array}{l}\text { Manejo de Información digital: Navegar, filtrar datos, evaluar } \\
\text { información, gestionar información, datos y contenidos. }\end{array}$ \\
\hline & & $\begin{array}{c}\text { Comunicar: Interactuar, compartir, participar como ciudadano } \\
\text { y colaborar mediante tecnología de la información. }\end{array}$ \\
\hline & & $\begin{array}{l}\text { Creación de contenido digital: Desarrollar, integrar y reelaborar } \\
\text { contenido digital. Copyrighty licencias programar. }\end{array}$ \\
\hline & & $\begin{array}{l}\text { Seguridad: Proteger los equipos, datos personales y privacidad. } \\
\text { Proteger salud y bienestar. Proteger medio ambiente. }\end{array}$ \\
\hline & & $\begin{array}{l}\text { Solución de Problemas: Resolver problemas técnicos } \\
\text { Identificar necesidades y respuestas tecnológicas. Uso creativo } \\
\text { de las tecnologías digitales. Identificar gapsen. }\end{array}$ \\
\hline
\end{tabular}

Fuente: adaptado de Aguerrevere, et al. (2020), Peiró y Soler (2020).

Mientras que, Peiró y Soler (2020) proponen ante el coronavirus, la necesidad de blindar al talento humano, mediante la preparación en las habilidades propias de su perfil profesional y en las competencias transversales que hacen posible la colaboración entre ellos, es decir, el trabajo en equipo, manejo de las nuevas tecnologías, gestión de las emociones, entre otros, a fin de obtener un desempeño eficaz, productivo y satisfactorio. Agregan los autores, la relevancia de la preparación en las destrezas digitales, pues, facilitan la adecuación del trabajador a las nuevas realidades, apoyándose en el modelo de competencias Digicomp formulado por la Unión Europea, el cual contempla el manejo de información de datos, comunicación, creación de contenido digital, seguridad y solución de problemas.

Resulta propicio aclarar que, el desarrollo de las competencias dependerá de la naturaleza de la organización, sus capacidades y necesidades. En efecto, cada empresa tiene una realidad específica, por tanto, la gerencia está llamada a repensar la actividad organizacional sobre la base de la formación por competencias como una herramienta favorable, capaz de aminorar los efectos provocados por la pandemia del COVID-19. Conviene destacar que, la formación por sí sola no garantiza el éxito, ésta deberá respaldarse con políticas que contribuyan a su normal desenvolvimiento y al logro de la productividad. 
En tal sentido, la OIT (2020a) sugiere ante el coronavirus, un conjunto de políticas a partir de las normas internacionales del trabajo, entre las cuales se encuentran apoyar a las empresas, al empleo y los ingresos, mediante la protección social a toda la población, aplicando medidas de mantenimiento del empleo y ofreciendo ayuda financiera/fiscal y otros medios de alivio. De este modo, se auxiliarán a los sectores más afectados, destinando recursos que estimulen el empleo, conservación de los puestos de trabajo, atención a la economía informal, garantía de protección social, entre otros. Aclarando que, cada país actuará de acuerdo a sus realidades y recursos.

En esta misma dirección, el Observatorio de la OIT (2020b) señaló cuatro pilares de actuación en el ámbito político, tales como estímulo de la economía y empleo, apoyo a las empresas, el empleo y los ingresos, protección en el lugar de trabajo, búsqueda de soluciones mediante el diálogo social, respaldo a los trabajadores de la economía informal, entre otros. Por tanto, los gobiernos están en la obligación de activarse en beneficio de las organizaciones, en aras de obtener un mejor porvenir para el mundo, en medio del impacto provocado por la pandemia de la COVID-19.

\section{Metodología}

La postura epistemológica en la cual se construyó la investigación fue el enfoque cualitativo, puesto que busca comprender e interpretar la realidad, bajo el ámbito de una investigación documental, entendida por Hurtado (2000) como un procedimiento científico de indagación, organización e interpretación de los datos e información alrededor de un tema en particular. Cabe agregar que, el diseño de la investigación fue documental, apoyándose en los aportes de la OIT (2020), Cejas et al. (2019), Aguerrevere et al. (2020), Peir y Soler (2020), entre otros. Al respecto, la data fue recopilada en libros, revistas digitales, partiendo de estudios y apreciaciones recientes en torno a la temática.

Es propicio mencionar el uso de las técnicas de análisis de contenido, a fin de conocer en detalle la información contenida en las fuentes consultadas. Para Barrera (2009), estas técnicas constituyen básicamente herramientas con propósitos analíticos e interpretativos, cuya aplicación depende de la capacidad relacional, conocimientos y disposición de ánimo. De esta manera, se llevó a cabo el análisis de las fuentes, interpretación de teorías, determinación de los hechos más relevantes, elaboración de mapas, entre otros.

\section{Resultados y discusión}

En base al conocimiento obtenido, derivado del análisis e interpretación de las fuentes, valiéndose de las técnicas de análisis de contenido, se obtuvo como resultado que, la formación por competencias es de gran relevancia, puesto que constituye una alternativa para que las organizaciones sean capaces de enfrentarse a los desafíos en el mundo laboral, provocados por la pandemia de la COVID-19. Ahora bien, los investigadores Aguerrevere,et al., (2020), Peir y Soler (2020) expresan un conjunto de habilidades que han de estar presentes en el talento humano, tales como las destrezas digitales, cognitivas, emocionales, manejo de las redes, resolución de conflictos, adaptación a los cambios, entre 
otros, lo cual contribuiría a la empleabilidad, alto rendimiento en los procesos, capacidad de responder a las realidades actuales, entre otros.

En tal sentido, las organizaciones han de contemplar los nuevos requerimientos del talento humano en el mundo laboral, por tanto, implementarán procesos formativos que estén soportados en herramientas tecnológicas, plataformas en líneas, redes sociales, whatsapp, correos electrónicos, canales youtube, entre otros, cuyos resultados se traducirían en el buen desempeño del personal. Sin embargo, las empresas necesitan estar respaldadas por un conjunto de medidas que les permitan subsistir y salir adelante en medio de las dificultades, provocadas por la pandemia del coronavirus.

Es así, como en estudios recientes, el Observatorio de la OIT (2020c) destacó los riesgos en el mercado laboral, por ende, los gobiernos proporcionarán soluciones integrales que enlacen el marco general de las políticas establecidas por la Organización Internacional del Trabajo para enfrentar el COVID-19. Al respecto, le otorga especial atención a la enseñanza y al desarrollo de las competencias, incluidos los conocimientos digitales y aprendizaje electrónico, capacitación profesional, iniciativa empresarial, protección social y la mejora de los derechos, entre otros. Lo anterior, revela la urgencia de formar al personal, apuntalando los esfuerzos de la gerencia al desarrollo de las competencias que, permitan potenciar el capital humano y así poder enfrentar los desafíos actuales.

\section{Consideraciones finales}

Los efectos económicos y sociales del COVID-19, han impactado el mercado laboral, conduciendo a las organizaciones a nuevas formas de trabajo, con plataformas virtuales, páginas web, automatización, teletrabajo, entre otros, con el propósito de formar al talento humano en base a las actuales circunstancias.

Por otra parte, la gerencia del talento humano ha de reflexionar sobre sus esquemas tradicionales, formular estrategias no solo para el presente; sino también para el futuro, apuntalando la mirada hacia la finalización de la pandemia. Por ende, está llamada a romper paradigmas, prepararse constantemente, fomentar el aprendizaje, ya que el camino es incierto y el éxito dependerá de las capacidades del personal. En tales escenarios, la formación por competencias constituye una vía que permite a las empresas la obtención de mayores rendimientos, ya que contribuye con el desarrollo del talento humano, adaptándose a las nuevas realidades del mercado de trabajo.

No obstante, el proceso formativo ha de estar acompañado por otras medidas que beneficien a las organizaciones, a fin de superar las dificultades que han surgido en el entorno laboral, producto del coronavirus. Por tanto, desde el escenario político se deberá proporcionar el apoyo tanto a los trabajadores como a las empresas, en especial a los sectores más afectados. En este sentido, cada gobierno actuará en función de sus recursos financieros, potencialidades de la economía, disponibilidad de financiamiento internacional, entre otros. 


\section{Referencias bibliográficas}

Aguerrevere, G., Amaral, N., Bentata, C. y Rucci, G. (2020). Desarrollo de habilidades para el mercado laboral en el contexto de la COVID-19. Recuperado de http: //publications.iadb.org/es/desarrollo-de-habilidades-para-el-mercado-laboralen-el-contexto-de-la-covid-19.

Alles, M. (2007). 5 Pasos para transformar una Oficina de personas en un Área de Recursos Humanos. Argentina. Granica, S.A

Alles, M. (2007). Gestión por Competencia. Diccionario. Argentina. Granica, S.A.

Alles, M. (2009). Construyendo talento. Programas de Desarrollo para el crecimiento de las personas y la continuidad en las organizaciones. Buenos aires. Argentina. Granica, SA.

Alles, M. (2017). Desarrollo del talento humano basado en competencias. España. Granica, S.A

Barrera, M. (2009). Técnicas de análisis en investigación. Caracas. Ediciones Quiron, SA.

Cejas, M., Rueda, M., Cayo, L. y Villa L. (2019). Formación por Competencias. Reto de la educación superior. Revista de Ciencias Sociales, 25(1), 1-8. Recuperado de https: //www.redalyc.org/jatsRepo/280/28059678009/28059678009.pdf.

Cejas, M. (2008). La Formación profesional basada en competencias. Consejo de Desarrollo Científico Humanístico. Valencia. Venezuela

Comisión Económica para América Latina y el Caribe (CEPAL) (2020). Informe especial COVID-19. Recuperado de: https://repositorio.cepal.org/bitstream/ handle/11362/45445/1/S2000286_es.pdf.

Delloitte (2020). COVID-19. Estrategias de la Fuerza Laboral para la Recuperación Post-COVID. Recuperado de https://www2.deloitte.com/content/dam/ Deloitte/cl/Documents/povs-covid19/Estrategias\%20de\%201a\%20Fuerza\%20 Laboral\%20para\%201a\%20Recuperaci\%C3\%B3n\%20Post-COVID.pdf.

Hurtado, J. (2000). Metodología de la Investigación Holística. Fundación Sypal. Caracas. Venezuela.

Maldonado, M. (2012). Programa de Formación por Competencias para el Talento Humano Adscrito al Departamento de Presupuesto de la UNEFM. (Tesis de Maestría). Universidad Nacional Experimental "Rafael María Baralt”. Coro. Venezuela.

Márquez, J. y Díaz, J. (2005). Formación del Recurso Humano por competencias. Recuperado de: http:www.redalyc.uaemex.mx/pdf/410/41060106.pdf.

Martínez, P. y Echeverría, B. (2009). Formación basada en Competencias. Revista de Investigación educativa, 27 (1), 125-147. Recuperado de https://www.redalyc. org/pdf/2833/283322804008.pdf. 
Organización Internacional del Trabajo (OIT) (2020a). El COVID-19 y el mundo del trabajo: Repercusiones y respuestas. Recuperado de https://www.ilo.org/ wcmsp5/groups/public/---dgreports/---dcomm/documents/briefingnote/ wcms_739158.pdf.

Organización Internacional Del Trabajo (OIT) (2020b). Observatorio de la OIT: El COVID-19 y el mundo del trabajo. Segunda edición. Estimaciones actualizadas y análisis. Recuperado de https:://www.oitcinterfor.org/node/7777.

Organización Internacional Del Trabajo (OIT) (2020c). Observatorio de la OIT: El COVID-19 y el mundo del trabajo. Cuarta edición. Estimaciones actualizadas y análisis. Recuperado de https://reliefweb.int/sites/reliefweb. int/files/resources/Observatorio $\% 20 \mathrm{de} \% 201 \mathrm{a} \% 20 \mathrm{OIT} \% 20 \% 20 \mathrm{E} 1 \% 20$ COVID\%E2\%80\%9119\%20y\%20el\%20mundo\%20del\%20trabajo.\%20 Cuarta\%20edici\%C3\%B3n.pdf

Peiró, J. y Soler, A. (2020). El Impulso al Teletrabajo Durante El Covid-19 y los Retos que Plantea. . Recuperado de https://www.ivie.es /wp-content/uploads/2020/05/11. covid19ivieExpress.El-impulso-al-teletrabajo-durante-el-COVID-19-y-losretos-que-plantea.pdf.

Tobón, S. (2012). Formación basada en competencias. Colombia. Ediciones ECO.

Valverde, O. (2001). El enfoque de la competencia laboral. Departamento de Publicaciones de Cinterfor/OIT. Uruguay.

Vela, L. (2004). Gestión por competencia. El reto compartido del crecimiento profesional y de la organización. Madrid. España. Esis Editorial.

Zapata, J. (2015). El modelo y enfoque de formación por competencias en la Educación Superior: Apuntes sobre sus fortalezas y debilidades. Revista Academia y Virtualidad, 8(2), 24-33. Recuperado de https://dialnet.unirioja.es/servlet/ articulo? codigo $=5236382$. 